\title{
Improved Stability Analysis for Neural Networks with Time-Varying Delay
}

\author{
Yongming Li, Junkang Tian, Jinzhou Zhao, \\ Liehui Zhang, and Tiejun Li
}

State Key Laboratory of Oil and Gas Reservoir Geology and Exploitation, Southwest Petroleum University, Chengdu, Sichuan 610500, China

Correspondence should be addressed to Junkang Tian, junkangtian@163.com

Received 20 February 2012; Accepted 30 March 2012

Academic Editor: Cristian Toma

Copyright (C 2012 Yongming Li et al. This is an open access article distributed under the Creative Commons Attribution License, which permits unrestricted use, distribution, and reproduction in any medium, provided the original work is properly cited.

This paper concerned the problem of delay-dependent asymptotic stability for neural networks with time-varying delay. A new class of Lyapunov functional dividing the interval delay is constructed to derive some new delay-dependent stability criteria. The obtained criteria are less conservative because free-weighting matrices method, a convex optimization approach, and a mixed dividing delay interval approach are considered. Finally, numerical examples are given to illustrate the effectiveness of the proposed method.

\section{Introduction}

In the past few decades, delayed neural networks have been investigated extensively because of their successful applications in various scientific areas, such as pattern recognition, image processing, associative memories, and parallel computation. It is well known that time delay is frequently encountered in neural networks, and it is often a major cause of instability and oscillation. Thus, the stability analysis of delayed neural networks has been widely considered by many research results, delay-independent ones [1-3], and delay-dependent ones [4-37]. Generally speaking, the delay-dependent stability criteria are less conservative than delay-independent ones when the time delay is small. Therefore, much attention has been paid to develop delay-dependent derived in [6] by considering some useful terms and using the free-weighting matrices method. By the fact that the neuron activation functions are sector bounded and nondecreasing, [7] presents an improved method, named the delay-slope-dependent method, for stability analysis of neural networks with time-varying delays. The method includes more information on the slope of neuron activation functions 
and fewer matrix variables in the constructing Lyapunov functionals. Then some new delay-dependent stability criteria with less conservatism are obtained. Recently, some new Lyapunov functionals based on the idea of decomposing the delay were introduced to investigate the stability of neural networks with time-invariant delay [10-12] and timevarying delay [13-16], which significantly reduced the conservativeness of the derived stability criteria. In [13], different from some previous results, the delay interval $[0, d(t)]$ is divided into some variable subintervals by employing weighting delays. Thus, some new delay-dependent stability criteria for neural networks with time varying delay are derived by applying the weighting-delay method, which are less conservative than the existing results. However, when the delay is time-varying, the information of subinterval is not considered sufficiently. For example, the time-varying delay $\tau(t)$ satisfies $0 \leqslant \tau(t) \leqslant h$. When the delay interval $[0, \tau(t)]$ is divided into some subintervals, the delay interval $[0, h]$ is also divided into some subintervals, in essence. But in the construction of Lyapunov functional in [15], this important information is ignored, which is a major source of conservativeness. Furthermore, the purpose of reducing conservatism is still limited due to the existence of multiple coefficients and the impact of subintervals with uniform size. Thus, it is still a quite difficult task to divide interval $[0, \tau(t)]$ in a more reasonable manner, so that the functional with the augmented matrix can easily be constructed to obtain less conservative stability results, which motivates our present study.

In this paper, the problem of delay-dependent asymptotic stability criterion for neural networks with time-varying delay has been considered. A new class of Lyapunov functional is constructed to derive some new delay-dependent stability criteria. The obtained criteria are less conservative because a mixed dividing delay interval approach is considered. Finally, the numerical examples are given to indicate significant improvements over some existing results.

\section{Problem Formulation}

Consider the following neural networks with time-varying delay:

$$
\dot{x}(t)=-C x(t)+A g(x(t))+B g(x(t-\tau(t)))+\mu,
$$

where $x(t)=\left[x_{1}(t), x_{2}(t), \ldots, x_{n}(t)\right]^{T} \in R^{n}$ is the neuron state vector, $g(x(\cdot))=\left[g_{1}\left(x_{1}(\cdot)\right)\right.$, $\left.g_{2}\left(x_{2}(\cdot)\right), \ldots, g_{n}\left(x_{n}(\cdot)\right)\right]^{T} \in R^{n}$ denotes the neuron activation function, and $\mu=\left(\mu_{1}, \mu_{2}, \ldots\right.$, $\left.\mu_{n}\right)^{T} \in \mathcal{R}^{n}$ is a constant input vector. $A, B \in \mathcal{R}^{n \times n}$ are the connection weight matrix and the delayed connection weight matrix, respectively. $C=\operatorname{diag}\left(C_{1}, C_{2}, \ldots, C_{n}\right)$ with $C_{i}>0, i=1$, $2, \ldots, n . \tau(t)$ is a time-varying continuous function that satisfies $0 \leqslant \tau(t) \leqslant h, \dot{\tau}(t) \leqslant u$, where $h$ and $u$ are constants. In addition, it is assumed that each neuron activation function in (2.1), $g_{i}(\cdot), i=1,2, \ldots, n$, is bounded and satisfies the following condition:

$$
0 \leqslant \frac{g_{i}(x)-g_{i}(y)}{x-y} \leqslant k_{i}, \quad \forall x, y \in R, x \neq y, i=1,2, \ldots, n
$$

where $k_{i}, i=1,2, \ldots, n$ are positive constants. 
Assuming that $x^{*}=\left[x_{1}^{*}, x_{2}^{*}, \ldots, x_{n}^{*}\right]^{T}$ is the equilibrium point of (2.1) whose uniqueness has been given in [31] and using the transformation $z(\cdot)=x(\cdot)-x^{*}$, system (2.1) can be converted to the following system:

$$
\dot{z}(t)=-C z(t)+A f(z(t))+B f(z(t-\tau(t)))
$$

where $z(t)=\left[z_{1}(t), z_{2}(t), \ldots, z_{n}(t)\right]^{T}, f(z(\cdot))=\left[f_{1}\left(z_{1}(\cdot)\right), f_{2}\left(z_{2}(\cdot)\right), \ldots, f_{n}\left(z_{n}(\cdot)\right)\right]^{T}$, and $f_{i}\left(z_{i}(\cdot)\right)=g_{i}\left(z_{i}(\cdot)+x_{i}^{*}\right)-g_{i}\left(x_{i}^{*}\right), i=1,2, \ldots, n$. According to the inequality (2.2), one can obtain that

$$
0 \leqslant \frac{f_{i}\left(z_{i}(t)\right)}{z_{i}(t)} \leqslant k_{i}, \quad f_{i}(0)=0, i=1,2, \ldots, n
$$

Thus, under this assumption, the following inequality holds for any diagonal matrix $Q>0$,

$$
z^{T}(t) K Q K z(t)-f^{T}(z(t)) Q f(z(t)) \geqslant 0
$$

where $K=\operatorname{diag}\left(k_{1}, k_{2}, \ldots, k_{n}\right)$.

Lemma 2.1 (see [38]). For any constant matrix $Z \in \mathcal{R}^{n \times n}, Z=Z^{T}>0$, scalars $h_{2}>h_{1}>0$, such that the following integrations are well defined, then

$$
-\left(h_{2}-h_{1}\right) \int_{t-h_{2}}^{t-h_{1}} x^{T}(s) Z x(s) d s \leqslant-\int_{t-h_{2}}^{t-h_{1}} x^{T}(s) d s Z \int_{t-h_{2}}^{t-h_{1}} x(s) d s
$$

\section{Main Results}

In this section, a new Lyapunov functional is constructed, and a new delay-dependent stability criterion is obtained.

Theorem 3.1. For given scalars $K=\operatorname{diag}\left(k_{1}, k_{2}, \ldots, k_{n}\right), h \geqslant 0, u$, and $0<\alpha<1$, the system (2.3) is globally asymptotically stable if there exist symmetric positive matrices $P=\left[P_{i j}\right]_{3 \times 3}, Q_{i}(i=$ $1,2, \ldots, 6), R_{i}(i=1,2, \ldots, 6)$, positive diagonal matrices $T_{1}, T_{2}, Q, \Delta=\operatorname{diag}\left(\delta_{1}, \delta_{2}, \ldots, \delta_{n}\right), \Lambda=$ $\operatorname{diag}\left(\lambda_{1}, \lambda_{2}, \ldots, \lambda_{n}\right)$, and any matrices $P_{1}, P_{2}, N_{i}, M_{i}, L_{i}, H_{i}, Z_{i}, S_{i}, U_{i}, V_{i}, W_{i}(i=1,2,3)$ with appropriate dimensions, such that the following LMIs hold:

$$
E_{1}=\left[\begin{array}{cccc}
E & \alpha h N & \frac{\alpha^{2} h^{2}}{2} H & \frac{\left(1-\alpha^{2}\right) h^{2}}{2} M \\
* & -\alpha h R_{3} & 0 & 0 \\
* & * & -\frac{\alpha^{2} h^{2}}{2} R_{5} & 0 \\
* & * & * & -\frac{\left(1-\alpha^{2}\right) h^{2}}{2} R_{6}
\end{array}\right]<0
$$




$$
\begin{gathered}
E_{2}=\left[\begin{array}{ccccc}
E & (1-\alpha) \alpha h L & \alpha^{2} h S & \frac{\alpha^{2} h^{2}}{2} H & \frac{\left(1-\alpha^{2}\right) h^{2}}{2} M \\
* & -(1-\alpha) \alpha h R_{3} & 0 & 0 & 0 \\
* & * & -\alpha^{2} h R_{3} & 0 & 0 \\
* & * & * & -\frac{\alpha^{2} h^{2}}{2} R_{5} & 0 \\
* & * & * & * & -\frac{\left(1-\alpha^{2}\right) h^{2}}{2} R_{6}
\end{array}\right]<0, \\
\Phi_{1}=\left[\begin{array}{ccccc}
\Phi & (1-\alpha) \alpha h U & (1-\alpha) h W & \frac{\alpha^{2} h^{2}}{2} H & \frac{\left(1-\alpha^{2}\right) h^{2}}{2} M \\
* & -(1-\alpha) \alpha h R_{3} & 0 & 0 & 0 \\
* & * & -(1-\alpha) h R_{4} & 0 & 0 \\
* & * & * & -\frac{\alpha^{2} h^{2}}{2} R_{5} & 0 \\
* & * & * & * & -\frac{\left(1-\alpha^{2}\right) h^{2}}{2} R_{6}
\end{array}\right]<0, \\
\Phi_{2}=\left[\begin{array}{ccccc}
\Phi & (1-\alpha) \alpha h V & (1-\alpha) h Z & \frac{\alpha^{2} h^{2}}{2} H & \frac{\left(1-\alpha^{2}\right) h^{2}}{2} M \\
* & -(1-\alpha) \alpha h R_{3} & 0 & 0 & 0 \\
* & * & -(1-\alpha) h R_{4} & 0 & 0 \\
* & * & * & -\frac{\alpha^{2} h^{2}}{2} R_{5} & 0 \\
* & * & * & * & -\frac{\left(1-\alpha^{2}\right) h^{2}}{2} R_{6}
\end{array}\right]<0,
\end{gathered}
$$

where

$$
E=\left(\begin{array}{ccccccccccc}
E_{11} & E_{12} & E_{13} & E_{14} & 0 & -P_{13} & E_{17} & P_{1} A+K T_{1} & P_{1} B & P_{22}-H_{1} & P_{23}-M_{1} \\
* & E_{22} & E_{23} & -N_{2} & 0 & 0 & 0 & 0 & 0 & -H_{2} & -M_{2} \\
* & * & E_{33} & -N_{3} & 0 & 0 & 0 & 0 & K T_{2} & -H_{3} & -M_{3} \\
* & * & * & E_{44} & 0 & \frac{R_{4}}{(1-\alpha) h} & 0 & 0 & 0 & -P_{22}+P_{23}^{T} & -P_{23}+P_{33} \\
* & * & * & * & -Q_{5} & 0 & 0 & 0 & 0 & 0 & 0 \\
* & * & * & * & * & E_{66} & 0 & 0 & 0 & -P_{23}^{T} & -P_{33} \\
* & * & * & * & * & * & E_{77} & P_{2} A+\Lambda-\Delta & P_{2} B & P_{12} & P_{13} \\
* & * & * & * & * & * & * & Q_{6}-2 T_{1}-Q & 0 & 0 & 0 \\
* & * & * & * & * & * & * & * & E_{99} & 0 & 0 \\
* & * & * & * & * & * & * & * & * & -R_{1} & 0 \\
* & * & * & * & * & * & * & * & * & * & -R_{2}
\end{array}\right),
$$




$$
\begin{aligned}
& \Phi=\left(\begin{array}{ccccccccccc}
\Phi_{11} & \Phi_{12} & \Phi_{13} & \Phi_{14} & V_{1}+\frac{R_{3}}{\alpha^{2} h} & -P_{13}-W_{1} & \Phi_{17} & P_{1} A+K T_{1} & P_{1} B & P_{22}-H_{1} & P_{23}-M_{1} \\
* & \Phi_{22} & \Phi_{23} & -U_{2}+Z_{2} & V_{2} & -W_{2} & 0 & 0 & 0 & -H_{2} & -M_{2} \\
* & * & \Phi_{33} & -U_{3}+Z_{3} & V_{3} & -W_{3} & 0 & 0 & K T_{2} & -H_{3} & -M_{3} \\
* & * & * & -Q_{3} & 0 & 0 & 0 & 0 & 0 & -P_{22}+P_{23}^{T} & -P_{23}+P_{33} \\
* & * & * & * & -Q_{5}-\frac{R_{3}}{\alpha^{2} h} & 0 & 0 & 0 & 0 & 0 & 0 \\
* & * & * & * & * & -Q_{4} & 0 & 0 & 0 & -P_{23}^{T} & -P_{33} \\
* & * & * & * & * & * & \Phi_{77} & P_{2} A+\Lambda-\Delta & P_{2} B & P_{12} & P_{13} \\
* & * & * & * & * & * & * & Q_{6}-2 T_{1}-Q & 0 & 0 & 0 \\
* & * & * & * & * & * & * & * & \Phi_{99} & 0 & 0 \\
* & * & * & * & * & * & * & * & * & -R_{1} & 0 \\
* & * & * & * & * & * & * & * & * & * & -R_{2}
\end{array}\right) \\
& E_{11}=P_{12}+P_{12}^{T}+Q_{1}+Q_{2}+Q_{3}+Q_{4}+Q_{5}+\alpha^{2} h^{2} R_{1}+(1-\alpha)^{2} h^{2} R_{2}+S_{1}+S_{1}^{T}-P_{1} C-C P_{1}^{T} \\
& +\alpha h\left(H_{1}+H_{1}^{T}\right)+(1-\alpha) h\left(M_{1}+M_{1}^{T}\right)+K Q K, \\
& E_{12}=L_{1}+S_{2}^{T}-S_{1}+\alpha h H_{2}^{T}+(1-\alpha) h M_{2}^{T} \text {, } \\
& E_{13}=N_{1}-L_{1}+S_{3}^{T}+\alpha h H_{3}^{T}+(1-\alpha) h M_{3}^{T} \text {, } \\
& E_{14}=-P_{12}+P_{13}-N_{1} \\
& E_{17}=P_{11}+K \Delta-P_{1}-C P_{2}^{T}, \\
& E_{22}=-(1-\alpha u) Q_{1}+L_{2}+L_{2}^{T}-S_{2}-S_{2}^{T} \text {, } \\
& E_{23}=-L_{2}+L_{3}^{T}-S_{3}^{T}+N_{2} \\
& E_{33}=-(1-u) Q_{2}+N_{3}+N_{3}^{T}-L_{3}-L_{3}^{T}-(1-u) K Q K, \\
& E_{44}=-Q_{3}-\frac{R_{4}}{(1-\alpha) h} \text {, } \\
& E_{66}=-Q_{4}-\frac{R_{4}}{(1-\alpha) h} \\
& E_{77}=\alpha h R_{3}+(1-\alpha) h R_{4}+\frac{\alpha^{2} h^{2}}{2} R_{5}+\frac{\left(1-\alpha^{2}\right) h^{2}}{2} R_{6}-P_{2}-P_{2}^{T}, \\
& E_{99}=-(1-u) Q_{6}-2 T_{2}+(1-u) Q \text {, } \\
& \Phi_{11}=P_{12}+P_{12}^{T}+Q_{1}+Q_{2}+Q_{3}+Q_{4}+Q_{5}+\alpha^{2} h^{2} R_{1}+(1-\alpha)^{2} h^{2} R_{2}-P_{1} C-C P_{1}^{T} \\
& +\alpha h\left(H_{1}+H_{1}^{T}\right)+(1-\alpha) h\left(M_{1}+M_{1}^{T}\right)-\frac{R_{3}}{\alpha^{2} h}+K Q K, \\
& \Phi_{12}=U_{1}-V_{1}+\alpha h H_{2}^{T}+(1-\alpha) h M_{2}^{T}, \\
& \Phi_{13}=W_{1}-Z_{1}+\alpha h H_{3}^{T}+(1-\alpha) h M_{3}^{T},
\end{aligned}
$$




$$
\begin{aligned}
& \Phi_{14}=-P_{12}+P_{13}-U_{1}+Z_{1}, \\
& \Phi_{17}=P_{11}+K \Delta-P_{1}-C P_{2}^{T}, \\
& \Phi_{22}=-(1-\alpha u) Q_{1}+U_{2}+U_{2}^{T}-V_{2}-V_{2}^{T}, \\
& \Phi_{23}=U_{3}^{T}-V_{3}^{T}+W_{2}-Z_{2}, \\
& \Phi_{33}=-(1-u) Q_{2}+W_{3}+W_{3}^{T}-Z_{3}-Z_{3}^{T}-(1-u) K Q K, \\
& \Phi_{77}=\alpha h R_{3}+(1-\alpha) h R_{4}+\frac{\alpha^{2} h^{2}}{2} R_{5}+\frac{\left(1-\alpha^{2}\right) h^{2}}{2} R_{6}-P_{2}-P_{2}^{T}, \\
& \Phi_{99}=-(1-u) Q_{6}-2 T_{2}+(1-u) Q, \\
& N=\left(\begin{array}{lllllllllll}
N_{1}^{T} & N_{2}^{T} & N_{3}^{T} & 0 & 0 & 0 & 0 & 0 & 0 & 0 & 0
\end{array}\right)^{T}, \\
& L=\left(\begin{array}{lllllllllll}
L_{1}^{T} & L_{2}^{T} & L_{3}^{T} & 0 & 0 & 0 & 0 & 0 & 0 & 0 & 0
\end{array}\right)^{T}, \\
& S=\left(\begin{array}{lllllllllll}
S_{1}^{T} & S_{2}^{T} & S_{3}^{T} & 0 & 0 & 0 & 0 & 0 & 0 & 0 & 0
\end{array}\right)^{T}, \\
& H=\left(\begin{array}{lllllllllll}
H_{1}^{T} & H_{2}^{T} & H_{3}^{T} & 0 & 0 & 0 & 0 & 0 & 0 & 0 & 0
\end{array}\right)^{T}, \\
& M=\left(\begin{array}{llllllllllll}
M_{1}^{T} & M_{2}^{T} & M_{3}^{T} & 0 & 0 & 0 & 0 & 0 & 0 & 0 & 0
\end{array}\right)^{T}, \\
& U=\left(\begin{array}{lllllllllll}
U_{1}^{T} & U_{2}^{T} & V_{3}^{T} & 0 & 0 & 0 & 0 & 0 & 0 & 0 & 0
\end{array}\right)^{T}, \\
& V=\left(\begin{array}{lllllllllll}
V_{1}^{T} & V_{2}^{T} & V_{3}^{T} & 0 & 0 & 0 & 0 & 0 & 0 & 0 & 0
\end{array}\right)^{T}, \\
& W=\left(\begin{array}{lllllllllll}
W_{1}^{T} & W_{2}^{T} & W_{3}^{T} & 0 & 0 & 0 & 0 & 0 & 0 & 0 & 0
\end{array}\right)^{T}, \\
& Z=\left(\begin{array}{lllllllllll}
Z_{1}^{T} & Z_{2}^{T} & Z_{3}^{T} & 0 & 0 & 0 & 0 & 0 & 0 & 0 & 0
\end{array}\right)^{T} .
\end{aligned}
$$

Proof. Construct a new class of Lyapunov functional candidate as follows:

$$
V(z(t))=\sum_{i=1}^{6} V_{i}(z(t))
$$

where

$$
\begin{aligned}
V_{1}(z(t))= & \xi^{T}(t) P \xi(t)+2 \sum_{i=1}^{n} \lambda_{i} \int_{0}^{z_{i}(t)} f_{i}(s) d s+2 \sum_{i=1}^{n} \delta_{i} \int_{0}^{z_{i}(t)}\left(k_{i} s-f_{i}(s)\right) d s, \\
V_{2}(z(t))= & \int_{t-\alpha \tau(t)}^{t} z^{T}(s) Q_{1} z(s) d s+\int_{t-\tau(t)}^{t} z^{T}(s) Q_{2} z(s) d s+\int_{t-\alpha h}^{t} z^{T}(s) Q_{3} z(s) d s \\
& +\int_{t-h}^{t} z^{T}(s) Q_{4} z(s) d s+\int_{t-\alpha^{2} h}^{t} z^{T}(s) Q_{5} z(s) d s+\int_{t-\tau(t)}^{t} f^{T}(z(s)) Q_{6} f(z(s)) d s,
\end{aligned}
$$




$$
\begin{aligned}
& V_{3}(z(t))=\alpha h \int_{-\alpha h}^{0} \int_{t+\theta}^{t} z^{T}(s) R_{1} z(s) d s d \theta+(1-\alpha) h \int_{-h}^{-\alpha h} \int_{t+\theta}^{t} z^{T}(s) R_{2} z(s) d s d \theta, \\
& V_{4}(z(t))=\int_{-\alpha h}^{0} \int_{t+\theta}^{t} \dot{z}^{T}(s) R_{3} \dot{z}(s) d s d \theta+\int_{-h}^{-\alpha h} \int_{t+\theta}^{t} \dot{z}^{T}(s) R_{4} \dot{z}(s) d s d \theta, \\
& V_{5}(z(t))=\int_{-\alpha h}^{0} \int_{\theta}^{0} \int_{t+\lambda}^{t} \dot{z}^{T}(s) R_{5} \dot{z}(s) d s d \lambda d \theta+\int_{-h}^{-\alpha h} \int_{\theta}^{0} \int_{t+\lambda}^{t} \dot{z}^{T}(s) R_{6} \dot{z}(s) d s d \lambda d \theta, \\
& V_{6}(z(t))=\int_{t-\tau(t)}^{t}\left[z^{T}(s) K Q K z(s)-f^{T}(z(s)) Q f(z(s))\right] d s,
\end{aligned}
$$

where

$$
\xi^{T}(t)=\left[z^{T}(t) \int_{t-\alpha h}^{t} z^{T}(s) d s \int_{t-h}^{t-\alpha h} z^{T}(s) d s\right], \quad 0<\alpha<1 .
$$

Remark 3.2. Since the term $2 \sum_{i=1}^{n} \delta_{i} \int_{0}^{z_{i}(t)}\left(k_{i} s-f_{i}(s)\right) d s$ in $V_{1}(z(t))$ and $V_{6}(z(t))=$ $\int_{t-\tau(t)}^{t}\left[z^{T}(s) K Q K z(s)-f^{T}(z(s)) Q f(z(s))\right] d s$ is taken into account, it is clear that the Lyapunov functional candidate in this paper is more general than that in $[5,6,8,9]$. So the stability criteria in this paper may be more applicable.

The time derivative of $V(z(t))$ along the trajectory of system (2.3) is given by

$$
\dot{V}(z(t))=\sum_{i=1}^{6} \dot{V}_{i}(z(t))
$$

where

$$
\begin{aligned}
\dot{V}_{1}(z(t))= & 2 \xi^{T}(t) P\left[\begin{array}{c}
\dot{z}(t) \\
z(t)-z(t-\alpha h) \\
z(t-\alpha h)-z(t-h)
\end{array}\right]+2 f^{T}(z(t)) \Lambda \dot{z}(t)+2(K z(t))-f(z(t))^{T} \Delta \dot{z}(t), \\
\dot{V}_{2}(z(t))= & z^{T}(t)\left(Q_{1}+Q_{2}+Q_{3}+Q_{4}+Q_{5}\right) z(t)+f^{T}(z(t)) Q_{6} f(z(t)) \\
& -(1-\alpha u) z^{T}(t-\alpha \tau(t)) Q_{1} z(t-\alpha \tau(t))-(1-u) z^{T}(t-\tau(t)) Q_{2} z(t-\tau(t)) \\
& -z^{T}(t-\alpha h) Q_{3} z(t-\alpha h)-z^{T}(t-h) Q_{4} z(t-h)-z^{T}\left(t-\alpha^{2} h\right) Q_{5} z\left(t-\alpha^{2} h\right) \\
& -(1-u) f^{T}(z(t-\tau(t))) Q_{6} f(z(t-\tau(t))) .
\end{aligned}
$$


Using Lemma 2.1, one can obtain that

$$
\begin{aligned}
\dot{V}_{3}(z(t))= & \alpha^{2} h^{2} z^{T}(t) R_{1} z(t)+(1-\alpha)^{2} h^{2} z^{T}(t) R_{2} z(t)-\alpha h \int_{t-\alpha h}^{t} z^{T}(s) R_{1} z(s) d s \\
& -(1-\alpha) h \int_{t-h}^{t-\alpha h} z^{T}(s) R_{2} z(s) d s \\
\leqslant & \alpha^{2} h^{2} z^{T}(t) R_{1} z(t)+(1-\alpha)^{2} h^{2} z^{T}(t) R_{2} z(t) \\
& -\left(\int_{t-\alpha h}^{t} z(s) d s\right)^{T} R_{1}\left(\int_{t-\alpha h}^{t} z(s) d s\right)-\left(\int_{t-h}^{t-\alpha h} z(s) d s\right)^{T} R_{2}\left(\int_{t-h}^{t-\alpha h} z(s) d s\right), \\
\dot{V}_{4}(z(t))= & \dot{z}^{T}(t)\left(\alpha h R_{3}+(1-\alpha) h R_{4}\right) \dot{z}(t)-\int_{t-\alpha h}^{t} \dot{z}^{T}(s) R_{3} \dot{z}(s) d s-\int_{t-h}^{t-\alpha h} \dot{z}^{T}(s) R_{4} \dot{z}(s) d s, \\
\dot{V}_{5}(z(t))= & \dot{z}^{T}(t)\left(\frac{\alpha^{2} h^{2}}{2} R_{5}+\frac{\left(1-\alpha^{2}\right) h^{2}}{2} R_{6}\right) \dot{z}(t)-\int_{-\alpha h}^{0} \int_{t+\theta}^{t} \dot{z}^{T}(s) R_{5} \dot{z}(s) d s d \theta \\
& -\int_{-h}^{-\alpha h} \int_{t+\theta}^{t} \dot{z}^{T}(s) R_{6} \dot{z}(s) d s d \theta, \\
\dot{V}_{6}(z(t)) \leqslant & z^{T}(t) K Q K z(t)-f^{T}(z(t)) Q f(z(t))-(1-u) z^{T}(t-\tau(t)) K Q K z(t-\tau(t)) \\
& +(1-u) f^{T}(z(t-\tau(t))) Q f(z(t-\tau(t))) .
\end{aligned}
$$

(1) For the case of $0 \leqslant \tau(t) \leqslant \alpha h$, then it gets

$$
\begin{aligned}
-\int_{t-\alpha h}^{t} \dot{z}^{T}(s) R_{3} \dot{z}(s) d s= & -\int_{t-\alpha h}^{t-\tau(t)} \dot{z}^{T}(s) R_{3} \dot{z}(s) d s-\int_{t-\tau(t)}^{t-\alpha \tau(t)} \dot{z}^{T}(s) R_{3} \dot{z}(s) d s \\
& -\int_{t-\alpha \tau(t)}^{t} \dot{z}^{T}(s) R_{3} \dot{z}(s) d s .
\end{aligned}
$$

Similar to [8], the following equalities hold:

$$
\begin{aligned}
& 2 \zeta^{T}(t) N\left[z(t-\tau(t))-z(t-\alpha h)-\int_{t-\alpha h}^{t-\tau(t)} \dot{z}(s) d s\right]=0, \\
& 2 \zeta^{T}(t) L\left[z(t-\alpha \tau(t))-z(t-\tau(t))-\int_{t-\tau(t)}^{t-\alpha \tau(t)} \dot{z}(s) d s\right]=0, \\
& 2 \zeta^{T}(t) S\left[z(t)-z(t-\alpha \tau(t))-\int_{t-\alpha \tau(t)}^{t} \dot{z}(s) d s\right]=0, \\
& 2\left[z^{T}(t) P_{1}+\dot{z}^{T}(t) P_{2}\right][-\dot{z}(t)-C z(t)+A f(z(t))+B f(z(t-\tau(t)))]=0,
\end{aligned}
$$




$$
\begin{aligned}
& 2 \zeta^{T}(t) H\left[\alpha h z(t)-\int_{t-\alpha h}^{t} z(s) d s-\int_{-\alpha h}^{0} \int_{t+\theta}^{t} \dot{z}(s) d s d \theta\right]=0, \\
& 2 \zeta^{T}(t) M\left[(1-\alpha) h z(t)-\int_{t-h}^{t-\alpha h} z(s) d s-\int_{-h}^{-\alpha h} \int_{t+\theta}^{t} \dot{z}(s) d s d \theta\right]=0, \\
& \zeta^{T}(t)=\left[\begin{array}{lllllll}
z^{T}(t) & z^{T}(t-\alpha \tau(t)) & z^{T}(t-\tau(t)) & z^{T}(t-\alpha h) & z^{T}\left(t-\alpha^{2} h\right) & z^{T}(t-h) & \dot{z}^{T}(t)
\end{array}\right. \\
& \left.f^{T}(z(t)) \quad f^{T}(z(t-\tau(t))) \quad \int_{t-\alpha h}^{t} z^{T}(s) d s \quad \int_{t-h}^{t-\alpha h} z^{T}(s) d s\right] .
\end{aligned}
$$

It is easy to see that

$$
\begin{aligned}
& -2 \zeta^{T}(t) N \int_{t-\alpha h}^{t-\tau(t)} \dot{z}(s) d s \leqslant(\alpha h-\tau(t)) \zeta^{T}(t) N R_{3}^{-1} N^{T} \zeta(t)+\int_{t-\alpha h}^{t-\tau(t)} \dot{z}^{T}(s) R_{3} \dot{z}(s) d s \\
& -2 \zeta^{T}(t) L \int_{t-\tau(t)}^{t-\alpha \tau(t)} \dot{z}(s) d s \leqslant(1-\alpha) \tau(t) \zeta^{T}(t) L R_{3}^{-1} L^{T} \zeta(t)+\int_{t-\tau(t)}^{t-\alpha \tau(t)} \dot{z}^{T}(s) R_{3} \dot{z}(s) d s \\
& -2 \zeta^{T} S \int_{t-\alpha \tau(t)}^{t} \dot{z}(s) d s \leqslant \alpha \tau(t) \zeta^{T}(t) S R_{3}^{-1} S^{T} \zeta(t)+\int_{t-\alpha \tau(t)}^{t} \dot{z}^{T}(s) R_{3} \dot{z}(s) d s \\
& -2 \zeta^{T} H \int_{-\alpha h}^{0} \int_{t+\theta}^{t} \dot{z}(s) d s d \theta \leqslant \frac{\alpha^{2} h^{2}}{2} \zeta^{T}(t) H R_{5}^{-1} H^{T} \zeta(t)+\int_{-\alpha h}^{0} \int_{t+\theta}^{t} \dot{z}^{T}(s) R_{5} \dot{z}(s) d s d \theta \\
& -2 \zeta^{T} M \int_{-h}^{-\alpha h} \int_{t+\theta}^{t} \dot{z}(s) d s d \theta \leqslant \frac{\left(1-\alpha^{2}\right) h^{2}}{2} \zeta^{T}(t) M R_{6}^{-1} M^{T} \zeta(t)+\int_{-h}^{-\alpha h} \int_{t+\theta}^{t} \dot{z}^{T}(s) R_{6} \dot{z}(s) d s d \theta .
\end{aligned}
$$

Using Lemma 2.1, it is easy to obtain that

$$
-\int_{t-h}^{t-\alpha h} \dot{z}^{T}(s) R_{4} \dot{z}(s) d s \leqslant-[z(t-\alpha h)-z(t-h)]^{T} \frac{R_{4}}{(1-\alpha) h}[z(t-\alpha h)-z(t-h)] .
$$

Furthermore, there exist positive diagonal matrices $T_{1}, T_{2}$, such that the following inequalities hold based on (2.4):

$$
\begin{gathered}
-2 f^{T}(z(t)) T_{1} f(z(t))+2 z^{T}(t) K T_{1} f(z(t)) \geqslant 0, \\
-2 f^{T}(z(t-\tau(t))) T_{2} f(z(t-\tau(t)))+2 z^{T}(t-\tau(t)) K T_{2} f(z(t-\tau(t))) \geqslant 0 .
\end{gathered}
$$


From (3.10)-(3.27), one can obtain that

$$
\dot{V}(z(t)) \leqslant \zeta^{T}(t) \Sigma_{1} \zeta(t)
$$

where

$$
\begin{aligned}
\Sigma_{1}= & E+(\alpha h-\tau(t)) N R_{3}^{-1} N^{T}+(1-\alpha) \tau(t) L R_{3}^{-1} L^{T}+\alpha \tau(t) S R_{3}^{-1} S^{T} \\
& +\frac{\alpha^{2} h^{2}}{2} H R_{5}^{-1} H^{T}+\frac{\left(1-\alpha^{2}\right) h^{2}}{2} M R_{6}^{-1} M^{T}
\end{aligned}
$$

Note that $0 \leqslant \tau(t) \leqslant \alpha h,(\alpha h-\tau(t)) N R_{3}^{-1} N^{T}+(1-\alpha) \tau(t) L R_{3}^{-1} L^{T}+\alpha \tau(t) S R_{3}^{-1} S^{T}$ can be seen as the convex combination of $N R_{3}^{-1} N^{T}, L R_{3}^{-1} L^{T}$, and $S R_{3}^{-1} S^{T}$ on $\tau(t)$. Therefore, $\Sigma_{1}<0$ holds if and only if

$$
\begin{gathered}
E+\alpha h N R_{3}^{-1} N^{T}+\frac{\alpha^{2} h^{2}}{2} H R_{5}^{-1} H^{T}+\frac{\left(1-\alpha^{2}\right) h^{2}}{2} M R_{6}^{-1} M^{T}<0, \\
E+(1-\alpha) \alpha h L R_{3}^{-1} L^{T}+\alpha^{2} h S R_{3}^{-1} S^{T}+\frac{\alpha^{2} h^{2}}{2} H R_{5}^{-1} H^{T}+\frac{\left(1-\alpha^{2}\right) h^{2}}{2} M R_{6}^{-1} M^{T}<0 .
\end{gathered}
$$

Applying the Schur complement, the inequalities (3.30) and (3.31) are equivalent to the LMI (3.1) and (3.2), respectively.

(2) When $\alpha h \leqslant \tau(t) \leqslant h$, then it gets

$$
\begin{aligned}
-\int_{t-\alpha h}^{t} \dot{z}^{T}(s) R_{3} \dot{z}(s) d s= & -\int_{t-\alpha h}^{t-\alpha \tau(t)} \dot{z}^{T}(s) R_{3} \dot{z}(s) d s-\int_{t-\alpha \tau(t)}^{t-\alpha^{2} h} \dot{z}^{T}(s) R_{3} \dot{z}(s) d s \\
& -\int_{t-\alpha^{2} h}^{t} \dot{z}^{T}(s) R_{3} \dot{z}(s) d s, \\
-\int_{t-h}^{t-\alpha h} \dot{z}^{T}(s) R_{4} \dot{z}(s) d s=- & -\int_{t-h}^{t-\tau(t)} \dot{z}^{T}(s) R_{4} \dot{z}(s) d s-\int_{t-\tau(t)}^{t-\alpha h} \dot{z}^{T}(s) R_{4} \dot{z}(s) d s .
\end{aligned}
$$

Similar to [8], the following equalities hold:

$$
\begin{gathered}
2 \zeta^{T}(t) U\left[z(t-\alpha \tau(t))-z(t-\alpha h)-\int_{t-\alpha h}^{t-\alpha \tau(t)} \dot{z}(s) d s\right]=0, \\
2 \zeta^{T}(t) V\left[z\left(t-\alpha^{2} h\right)-z(t-\alpha \tau(t))-\int_{t-\alpha \tau(t)}^{t-\alpha^{2} h} \dot{z}(s) d s\right]=0, \\
2 \zeta^{T}(t) W\left[z(t-\tau(t))-z(t-h)-\int_{t-h}^{t-\tau(t)} \dot{z}(s) d s\right]=0, \\
2 \zeta^{T}(t) Z\left[z(t-\alpha h)-z(t-\tau(t))-\int_{t-\tau(t)}^{t-\alpha h} \dot{z}(s) d s\right]=0 .
\end{gathered}
$$


It is easy to obtain that

$$
\begin{aligned}
& -2 \zeta^{T}(t) U \int_{t-\alpha h}^{t-\alpha \tau(t)} \dot{z}(s) \mathrm{d} s \leqslant \alpha(h-\tau(t)) \zeta^{T}(t) U R_{3}^{-1} U^{T} \zeta(t)+\int_{t-\alpha h}^{t-\alpha \tau(t)} \dot{z}^{T}(s) R_{3} \dot{z}(s) d s, \\
& -2 \zeta^{T}(t) V \int_{t-\alpha \tau(t)}^{t-\alpha^{2} h} \dot{z}(s) \mathrm{d} s \leqslant \alpha(\tau(t)-\alpha h) \zeta^{T}(t) V R_{3}^{-1} V^{T} \zeta(t)+\int_{t-\alpha \tau(t)}^{t-\alpha^{2} h} \dot{z}^{T}(s) R_{3} \dot{z}(s) d s, \\
& -2 \zeta^{T}(t) W \int_{t-h}^{t-\tau(t)} \dot{z}(s) \mathrm{d} s \leqslant(h-\tau(t)) \zeta^{T}(t) W R_{4}^{-1} W^{T} \zeta(t)+\int_{t-h}^{t-\tau(t)} \dot{z}^{T}(s) R_{4} \dot{z}(s) d s, \\
& -2 \zeta^{T}(t) Z \int_{t-\tau(t)}^{t-\alpha h} \dot{z}(s) \mathrm{d} s \leqslant(\tau(t)-\alpha h) \zeta^{T}(t) Z R_{4}^{-1} Z^{T} \zeta(t)+\int_{t-\tau(t)}^{t-\alpha h} \dot{z}^{T}(s) R_{4} \dot{z}(s) d s .
\end{aligned}
$$

Using Lemma 2.1, one can obtain that

$$
-\int_{t-\alpha^{2} h}^{t} \dot{z}^{T}(s) R_{4} \dot{z}(s) \mathrm{d} s \leqslant-\frac{1}{\alpha^{2} h}\left[z(t)-z\left(t-\alpha^{2} h\right)\right]^{T} R_{3}\left[z(t)-z\left(t-\alpha^{2} h\right)\right] .
$$

From (3.10)-(3.11), (3)-(3.18), (3.23), (3.24), (3.26), (3.27) and (3.33)-(3.35), one can obtain that

$$
\dot{V}(z(t)) \leqslant \zeta^{T}(t) \Sigma_{2} \zeta(t)
$$

where

$$
\begin{aligned}
\Sigma_{2}= & \Phi+\alpha(h-\tau(t)) U R_{3}^{-1} U^{T}+\alpha(\tau(t)-\alpha h) V R_{3}^{-1} V^{T}+(h-\tau(t)) W R_{4}^{-1} W^{T} \\
& +(\tau(t)-\alpha h) Z R_{4}^{-1} Z^{T}+\frac{\alpha^{2} h^{2}}{2} H R_{5}^{-1} H^{T}+\frac{\left(1-\alpha^{2}\right) h^{2}}{2} M R_{6}^{-1} M^{T}
\end{aligned}
$$

Note that $\alpha h \leqslant \tau(t) \leqslant h, \alpha(h-\tau(t)) U R_{3}^{-1} U^{T}+\alpha(\tau(t)-\alpha h) V R_{3}^{-1} V^{T}+(h-\tau(t)) W R_{4}^{-1} W^{T}+$ $(\tau(t)-\alpha h) Z R_{4}^{-1} Z^{T}$ can be seen as the convex combination of $U R_{3}^{-1} U^{T}, V R_{3}^{-1} V^{T}, W R_{4}^{-1} W^{T}$, and $Z R_{4}^{-1} Z^{T}$ on $\tau(t)$. Therefore, $\Sigma_{2}<0$ holds if and only if

$$
\begin{aligned}
& \Phi+\alpha(1-\alpha) h U R_{3}^{-1} U^{T}+(1-\alpha) h W R_{4}^{-1} W^{T}+\frac{\alpha^{2} h^{2}}{2} H R_{5}^{-1} H^{T}+\frac{\left(1-\alpha^{2}\right) h^{2}}{2} M R_{6}^{-1} M^{T}<0 \\
& \Phi+\alpha(1-\alpha) h V R_{3}^{-1} V^{T}+(1-\alpha) h Z R_{4}^{-1} Z^{T}+\frac{\alpha^{2} h^{2}}{2} H R_{5}^{-1} H^{T}+\frac{\left(1-\alpha^{2}\right) h^{2}}{2} M R_{6}^{-1} M^{T}<0 .
\end{aligned}
$$

Applying the Schur complement, the inequalities (3.38) and (3.39) are equivalent to the LMI (3.3) and (3.4), respectively. Therefore, if the LMIs (3.1)-(3.4) are satisfied, then the system (2.3) is guaranteed to be asymptotically stable for $0 \leqslant \tau(t) \leqslant h$. 
Remark 3.3. It is well known that the delay-dividing approach can reduce the conservatism notably. But some previous literature only uses single method to divide the delay interval $[0, h]$. Unlike $[10,25]$, the new Lyapunov functional in our paper which not only divides the delay interval $[0, h]$ into two ones $[0, \alpha h]$ and $[\alpha h, h]$ but also divides the delay interval $[0, h]$ into three ones $[0, \alpha \tau(t)],[\alpha \tau(t), \tau(t)]$, and $[\tau(t), h]$ is proposed. Each segment has a different positive matrix, which has the potential to yield less conservative results.

Remark 3.4. In this paper, by taking the states $z(t-\alpha h), z(t-\tau(t)), z\left(t-\alpha^{2} h\right), z(t-h)$, and $z(t-\alpha \tau(t))$ as augmented variables, the stability in Theorem 3.1 utilizes more information on state variables. And in deriving upper bounds of integral terms in $\dot{V}_{4}(z(t))$, different freeweighting matrices are introduced in two different intervals $0 \leqslant \tau(t) \leqslant \alpha h$ and $\alpha h \leqslant \tau(t) \leqslant h$. These methods mentioned above may lead to obtain an improved feasible region for delaydependent stability criteria.

Remark 3.5. In (3.28), $E+(\alpha h-\tau(t)) N R_{3}^{-1} N^{T}+(1-\alpha) \tau(t) L R_{3}^{-1} L^{T}+\alpha \tau(t) S R_{3}^{-1} S^{T}+$ $\left(\alpha^{2} h^{2} / 2\right) H R_{5}^{-1} H^{T}+\left(\left(1-\alpha^{2}\right) h^{2} / 2\right) M R_{6}^{-1} M^{T}$ is not simply guaranteed by $E+\alpha h N R_{3}^{-1} N^{T}+$ $(1-\alpha) \alpha h L R_{3}^{-1} L^{T}+\alpha^{2} h S R_{3}^{-1} S^{T}+\left(\alpha^{2} h^{2} / 2\right) H R_{5}^{-1} H^{T}+\left(\left(1-\alpha^{2}\right) h^{2} / 2\right) M R_{6}^{-1} M^{T}$ but is evaluated by the LMIs (3.1) and (3.2), which can help reduce much more conservatism than the results in [8].

Remark 3.6. In many cases, $u$ is unknown. For this situation, a rate-independent criterion for a delay satisfying $0 \leq \tau(t) \leq h$ is derived as follows by setting $Q_{1}=0, Q_{2}=0, Q_{6}=0$, and $Q=0$ in the proof of Theorem 3.1.

Corollary 3.7. For given scalars $K=\operatorname{diag}\left(k_{1}, k_{2}, \ldots, k_{n}\right), h \geqslant 0$, and $0<\alpha<1$, the system (2.3) is globally asymptotically stable if there exist symmetric positive matrices $P=\left[P_{i j}\right]_{3 \times 3}, Q_{3}, Q_{4}, Q_{5}$, $R_{i}(i=1,2, \ldots, 6)$, positive diagonal matrices $T_{1}, T_{2}, \Lambda=\operatorname{diag}\left(\lambda_{1}, \lambda_{2}, \ldots, \lambda_{n}\right), \Delta=$ $\operatorname{diag}\left(\delta_{1}, \delta_{2}, \ldots, \delta_{n}\right)$, and any matrices $P_{1}, P_{2}, N_{i}, M_{i}, L_{i}, H_{i}, Z_{i}, S_{i}, U_{i}, V_{i}, W_{i}(i=1,2,3)$ with appropriate dimensions, such that the following LMIs hold:

$$
\begin{aligned}
& \bar{E}_{1}=\left[\begin{array}{ccccc}
\bar{E} & \alpha h N & \frac{\alpha^{2} h^{2}}{2} H & \frac{\left(1-\alpha^{2}\right) h^{2}}{2} M \\
* & -\alpha h R_{3} & 0 & 0 \\
* & * & -\frac{\alpha^{2} h^{2}}{2} R_{5} & 0 \\
* & * & * & -\frac{\left(1-\alpha^{2}\right) h^{2}}{2} R_{6}
\end{array}\right]<0, \\
& \bar{E}_{2}=\left[\begin{array}{ccccc}
\bar{E} & (1-\alpha) \alpha h L & \alpha^{2} h S & \frac{\alpha^{2} h^{2}}{2} H & \frac{\left(1-\alpha^{2}\right) h^{2}}{2} M \\
* & -(1-\alpha) \alpha h R_{3} & 0 & 0 & 0 \\
* & * & -\alpha^{2} h R_{3} & 0 & 0 \\
* & * & * & -\frac{\alpha^{2} h^{2}}{2} R_{5} & 0 \\
* & * & * & * & -\frac{\left(1-\alpha^{2}\right) h^{2}}{2} R_{6}
\end{array}\right]<0,
\end{aligned}
$$


Mathematical Problems in Engineering

$$
\begin{gathered}
\bar{\Phi}_{1}=\left[\begin{array}{ccccc}
\bar{\Phi} & (1-\alpha) \alpha h U & (1-\alpha) h W & \frac{\alpha^{2} h^{2}}{2} H & \frac{\left(1-\alpha^{2}\right) h^{2}}{2} M \\
* & -(1-\alpha) \alpha h R_{3} & 0 & 0 & 0 \\
* & * & -(1-\alpha) h R_{4} & 0 & 0 \\
* & * & * & -\frac{\alpha^{2} h^{2}}{2} R_{5} & 0 \\
* & * & * & * & -\frac{\left(1-\alpha^{2}\right) h^{2}}{2} R_{6}
\end{array}\right]<0, \\
\bar{\Phi}_{2}=\left[\begin{array}{ccccc}
* & (1-\alpha) \alpha h V & (1-\alpha) h Z & \frac{\alpha^{2} h^{2}}{2} H & \frac{\left(1-\alpha^{2}\right) h^{2}}{2} M \\
* & -(1-\alpha) \alpha h R_{3} & 0 & 0 & 0 \\
* & * & -(1-\alpha) h R_{4} & 0 & 0 \\
* & * & * & -\frac{\alpha^{2} h^{2}}{2} R_{5} & 0 \\
* & * & * & -\frac{\left(1-\alpha^{2}\right) h^{2}}{2} R_{6}
\end{array}\right]<0,
\end{gathered}
$$

where

$$
\bar{E}=\left(\begin{array}{ccccccccccc}
\bar{E}_{11} & E_{12} & E_{13} & E_{14} & 0 & -P_{13} & E_{17} & P_{1} A+K T_{1} & P_{1} B & P_{22}-H_{1} & P_{23}-M_{1} \\
* & E_{22} & E_{23} & -N_{2} & 0 & 0 & 0 & 0 & 0 & -H_{2} & -M_{2} \\
* & * & E_{33} & -N_{3} & 0 & 0 & 0 & 0 & K T_{2} & -H_{3} & -M_{3} \\
* & * & * & E_{44} & 0 & \frac{R_{4}}{(1-\alpha) h} & 0 & 0 & 0 & -P_{22}+P_{23}^{T} & -P_{23}+P_{33} \\
* & * & * & * & -Q_{5} & 0 & 0 & 0 & 0 & 0 & 0 \\
* & * & * & * & * & E_{66} & 0 & 0 & 0 & -P_{23}^{T} & -P_{33} \\
* & * & * & * & * & * & E_{77} & P_{2} A+\Lambda-\Delta & P_{2} B & P_{12} & P_{13} \\
* & * & * & * & * & * & * & -2 T_{1} & 0 & 0 & 0 \\
* & * & * & * & * & * & * & * & -2 T_{2} & 0 & 0 \\
* & * & * & * & * & * & * & * & * & -R_{1} & 0 \\
* & * & * & * & * & * & * & * & * & * & -R_{2}
\end{array}\right),
$$$$
\bar{\Phi}=\left(\begin{array}{ccccccccccc}
\bar{\Phi}_{11} & \Phi_{12} & \Phi_{13} & \Phi_{14} & V_{1}+\frac{R_{3}}{\alpha^{2} h} & -P_{13}-W_{1} & \Phi_{17} & P_{1} A+K T_{1} & P_{1} B & P_{22}-H_{1} & P_{23}-M_{1} \\
* & \Phi_{22} & \Phi_{23} & -U_{2}+Z_{2} & V_{2} & -W_{2} & 0 & 0 & 0 & -H_{2} & -M_{2} \\
* & * & \Phi_{33} & -U_{3}+Z_{3} & V_{3} & -W_{3} & 0 & 0 & K T_{2} & -H_{3} & -M_{3} \\
* & * & * & -Q_{3} & 0 & 0 & 0 & 0 & 0 & -P_{22}+P_{23}^{T} & -P_{23}+P_{33} \\
* & * & * & * & -Q_{5}-\frac{R_{3}}{\alpha^{2} h} & 0 & 0 & 0 & 0 & 0 & 0 \\
* & * & * & * & * & -Q_{4} & 0 & 0 & 0 & -P_{23}^{T} & -P_{33} \\
* & * & * & * & * & * & \Phi_{77} & P_{2} A+\Lambda-\Delta & P_{2} B & P_{12} & P_{13} \\
* & * & * & * & * & * & * & -2 T_{1} & 0 & 0 & 0 \\
* & * & * & * & * & * & * & * & -2 T_{2} & 0 & 0 \\
* & * & * & * & * & * & * & * & * & -R_{1} & 0 \\
* & * & * & * & * & * & * & * & * & * & -R_{2}
\end{array}\right)
$$ 


$$
\begin{aligned}
\bar{E}_{11}= & P_{12}+P_{12}^{T}+Q_{3}+Q_{4}+Q_{5}+\alpha^{2} h^{2} R_{1}+(1-\alpha)^{2} h^{2} R_{2}+S_{1}+S_{1}^{T}-P_{1} C-C P_{1}^{T} \\
& \quad+\alpha h\left(H_{1}+H_{1}^{T}\right)+(1-\alpha) h\left(M_{1}+M_{1}^{T}\right) \\
\bar{E}_{22}= & L_{2}+L_{2}^{T}-S_{2}-S_{2}^{T}, \\
\bar{E}_{33}= & N_{3}+N_{3}^{T}-L_{3}-L_{3}^{T}, \\
\bar{\Phi}_{11}= & P_{12}+P_{12}^{T}+Q_{3}+Q_{4}+Q_{5}+\alpha^{2} h^{2} R_{1}+(1-\alpha)^{2} h^{2} R_{2}-P_{1} C-C P_{1}^{T} \\
& +\alpha h\left(H_{1}+H_{1}^{T}\right)+(1-\alpha) h\left(M_{1}+M_{1}^{T}\right)-\frac{R_{3}}{\alpha^{2} h}, \\
\bar{\Phi}_{22}= & U_{2}+U_{2}^{T}-V_{2}-V_{2}^{T}, \\
\bar{\Phi}_{33}= & W_{3}+W_{3}^{T}-Z_{3}-Z_{3}^{T} .
\end{aligned}
$$

The other $E_{i j}, \Phi_{i j}$ are defined in Theorem 3.1.

\section{Numerical Examples}

Example 4.1. Consider the stability of system (2.3) with time-varying delay and

$$
\begin{gathered}
C=\operatorname{diag}(2,2), \quad A=\left[\begin{array}{cc}
1 & 1 \\
-1 & -1
\end{array}\right], \quad B=\left[\begin{array}{cc}
0.88 & 1 \\
1 & 1
\end{array}\right], \quad \sigma_{1}=0.4, \\
\sigma_{2}=0.8, \quad \gamma_{1}=0, \quad \gamma_{2}=0 .
\end{gathered}
$$

Our purpose is to estimate the allowable upper bounds delay $h$ under different $u$ such that the system (2.3) is globally asymptotically stable. According to Table 1, this example shows that the stability criterion in this paper gives much less conservative results than those in the literature. By using the Matlab LMI toolbox, we solve LMIs (3.1)-(3.4) for the case $\alpha=0.4, u=$ 0.8 , and $h=2.9144$ and obtain

$$
\begin{gathered}
P_{11}=\left[\begin{array}{cc}
0.0005 & -0.0002 \\
-0.0002 & 0.0018
\end{array}\right], \quad P_{12}=1.0 e^{-003} \times\left[\begin{array}{cc}
-0.0363 & 0.0081 \\
0.1663 & -0.1945
\end{array}\right], \\
P_{13}=1.0 e^{-006} \times\left[\begin{array}{cc}
-0.1002 & 0.0252 \\
0.3993 & -0.2498
\end{array}\right], \quad P_{22}=1.0 e^{-004} \times\left[\begin{array}{cc}
0.1678 & -0.2152 \\
-0.2152 & 0.5047
\end{array}\right], \\
P_{23}=1.0 e^{-006} \times\left[\begin{array}{cc}
0.0221 & -0.0836 \\
0.0468 & -0.3688
\end{array}\right], \quad P_{33}=1.0 e^{-006} \times\left[\begin{array}{cc}
0.2614 & -0.2146 \\
-0.2146 & 0.5418
\end{array}\right],
\end{gathered}
$$


Mathematical Problems in Engineering

$$
\begin{aligned}
& Q_{1}=1.0 e^{-006} \times\left[\begin{array}{cc}
0.2368 & -0.2454 \\
-0.2454 & 0.4008
\end{array}\right], \quad Q_{2}=1.0 e^{-005} \times\left[\begin{array}{cc}
0.0174 & -0.0087 \\
-0.0087 & 0.1976
\end{array}\right] \text {, } \\
& Q_{3}=1.0 e^{-003} \times\left[\begin{array}{ll}
0.0526 & 0.0296 \\
0.0296 & 0.2541
\end{array}\right], \quad Q_{4}=1.0 e^{-004} \times\left[\begin{array}{ll}
0.9971 & 0.5110 \\
0.5110 & 0.4639
\end{array}\right], \\
& Q_{5}=1.0 e^{-005} \times\left[\begin{array}{cc}
0.0232 & -0.0561 \\
-0.0561 & 0.1584
\end{array}\right], \quad Q_{6}=\left[\begin{array}{ll}
0.0021 & 0.0035 \\
0.0035 & 0.0057
\end{array}\right], \\
& R_{1}=1.0 e^{-004} \times\left[\begin{array}{cc}
0.4523 & -0.6020 \\
-0.6020 & 0.8760
\end{array}\right], \quad R_{2}=1.0 e^{-006} \times\left[\begin{array}{cc}
0.1049 & -0.2159 \\
-0.2159 & 0.5299
\end{array}\right] \text {, } \\
& R_{3}=1.0 e^{-004} \times\left[\begin{array}{ll}
0.7915 & 0.3650 \\
0.3650 & 0.4110
\end{array}\right], \quad R_{4}=1.0 e^{-004} \times\left[\begin{array}{ll}
0.9709 & 0.5071 \\
0.5071 & 0.4212
\end{array}\right], \\
& R_{5}=1.0 e^{-003} \times\left[\begin{array}{cc}
0.1083 & -0.1872 \\
-0.1872 & 0.3776
\end{array}\right], \quad R_{6}=1.0 e^{-006} \times\left[\begin{array}{cc}
0.0698 & -0.1266 \\
-0.1266 & 0.2910
\end{array}\right] \text {, } \\
& \Lambda=\left[\begin{array}{cc}
0.0000 & 0 \\
0 & 0.0014
\end{array}\right], \quad \Delta=1.0 e^{-003} \times\left[\begin{array}{cc}
0.1686 & 0 \\
0 & 0.0027
\end{array}\right] \\
& T_{1}=\left[\begin{array}{cc}
0.0037 & 0 \\
0 & 0.0039
\end{array}\right], \quad T_{2}=\left[\begin{array}{cc}
0.0009 & 0 \\
0 & 0.0010
\end{array}\right], \quad Q=\left[\begin{array}{cc}
0.0000 & 0 \\
0 & 0.0038
\end{array}\right]
\end{aligned}
$$

Therefore, it follows from Theorem 3.1 that the system (2.3) with given parameters is globally asymptotically stable.

Example 4.2. Consider the stability of system (2.3) with time-varying delay and

$$
\begin{gathered}
C=\operatorname{diag}(1.5,0.7), \quad A=\left[\begin{array}{ll}
0.0503 & 0.0454 \\
0.0987 & 0.2075
\end{array}\right], \quad B=\left[\begin{array}{ll}
0.2381 & 0.9320 \\
0.0388 & 0.5062
\end{array}\right], \\
\sigma_{1}=0.3, \quad \sigma_{2}=0.8, \quad \gamma_{1}=0, \quad \gamma_{2}=0 .
\end{gathered}
$$

Table 2 gives the comparison results on the maximum delay bound allowed via the methods in recent paper and our new study. According to Table 2, this example shows that 
Table 1: Allowable upper bound of $h$ for different $u$ (Example 4.1).

\begin{tabular}{lccc}
\hline$u$ & $u=0.8$ & $u=0.9$ & $\begin{array}{c}\text { Unknown } \\
u\end{array}$ \\
\hline$[4,5]$ & 1.2281 & 0.8636 & 0.8298 \\
{$[6]$} & 1.6831 & 1.1493 & 1.0880 \\
{$[7]$} & 1.7347 & 1.1662 & - \\
{$[8]$} & 2.3534 & 1.6050 & 1.5103 \\
{$[13](\rho=0.8)$} & 2.5406 & 1.7273 & - \\
{$[14](m=2)$} & 2.2495 & 1.5966 & 1.4902 \\
{$[15](m=2)$} & 2.1150 & 1.4286 & 1.3126 \\
{$[15](m=3)$} & 2.3838 & 1.6229 & 1.4740 \\
This paper $(\alpha=0.4)$ & 2.9144 & 1.9095 & 1.7437 \\
This paper $(\alpha=0.5)$ & 2.9063 & 1.9443 & 1.7769 \\
\hline
\end{tabular}

Table 2: Allowable upper bound of $h$ for different $u$ (Example 4.2).

\begin{tabular}{lcccc}
\hline$u$ & $u=0.4$ & $u=0.45$ & $u=0.5$ & $u=0.55$ \\
\hline$[6]$ & 3.9972 & 3.2760 & 3.0594 & 2.9814 \\
{$[9]$} & 4.3814 & 3.6008 & 3.3377 & 3.2350 \\
This paper $(\alpha=0.4)$ & 4.7444 & 3.9482 & 3.7000 & 3.5667 \\
This paper $(\alpha=0.5)$ & 4.6587 & 3.9069 & 3.6496 & 3.5276 \\
\hline
\end{tabular}

the stability criterion in this paper can lead to less conservative results. By using the Matlab LMI toolbox, we solve LMIs (3.1)-(3.4) for the case $\alpha=0.4, u=0.4$, and $h=4.7444$ and obtain

$$
\begin{gathered}
P_{11}=\left[\begin{array}{cc}
1.0206 & -0.7528 \\
-0.7528 & 1.2378
\end{array}\right], \quad P_{12}=1.0 e^{-003} \times\left[\begin{array}{cc}
-0.0045 & 0.0417 \\
-0.0652 & -0.0137
\end{array}\right] \\
P_{13}=1.0 e^{-003} \times\left[\begin{array}{cc}
-0.2467 & 0.6404 \\
-0.1839 & -0.3921
\end{array}\right], \quad P_{22}=\left[\begin{array}{cc}
0.0073 & -0.0020 \\
-0.0020 & 0.0022
\end{array}\right], \\
P_{23}=1.0 e^{-004} \times\left[\begin{array}{cc}
-0.0086 & 0.4616 \\
-0.4108 & 0.1085
\end{array}\right], \quad P_{33}=1.0 e^{-003} \times\left[\begin{array}{cc}
0.1904 & -0.1292 \\
-0.1292 & 0.1059
\end{array}\right], \\
Q_{1}=\left[\begin{array}{cc}
0.0013 & -0.0012 \\
-0.0012 & 0.0012
\end{array}\right], \quad Q_{2}=\left[\begin{array}{cc}
0.0258 & 0.0566 \\
0.0566 & 0.1268
\end{array}\right], \quad Q_{3}=\left[\begin{array}{cc}
0.0653 & 0.0051 \\
0.0051 & 0.0050
\end{array}\right], \\
Q_{4}=\left[\begin{array}{cc}
0.0114 & -0.0300 \\
-0.0300 & 0.0839
\end{array}\right], \quad Q_{5}=\left[\begin{array}{cc}
0.0014 & -0.0009 \\
-0.0009 & 0.0006
\end{array}\right], \quad Q_{6}=\left[\begin{array}{cc}
0.0340 & 0.0300 \\
0.0300 & 0.0310
\end{array}\right], \\
R_{1}=\left[\begin{array}{cc}
0.0090 & -0.0066 \\
-0.0066 & 0.0062
\end{array}\right], \quad R_{2}=1.0 e^{-003} \times\left[\begin{array}{cc}
0.1943 & -0.1361 \\
-0.1361 & 0.1036
\end{array}\right],
\end{gathered}
$$




$$
\begin{gathered}
R_{3}=\left[\begin{array}{cc}
0.2485 & -0.3846 \\
-0.3846 & 0.7348
\end{array}\right], \quad R_{4}=\left[\begin{array}{cc}
0.0802 & -0.2197 \\
-0.2197 & 0.6122
\end{array}\right], \\
R_{5}=\left[\begin{array}{cc}
0.1553 & -0.0821 \\
-0.0821 & 0.0709
\end{array}\right], \quad R_{6}=1.0 e^{-003} \times\left[\begin{array}{cc}
0.7651 & -0.4906 \\
-0.4906 & 0.5825
\end{array}\right], \\
\Lambda=\left[\begin{array}{cc}
0.0318 & 0 \\
0 & 0.0344
\end{array}\right], \quad \Delta=1.0 e^{-003} \times\left[\begin{array}{cc}
0.0797 & 0 \\
0 & 0.3593
\end{array}\right], \\
T_{1}=\left[\begin{array}{cc}
0.0136 & 0 \\
0 & 0.1968
\end{array}\right], \quad T_{2}=\left[\begin{array}{cc}
0.3056 & 0 \\
0 & 0.3423
\end{array}\right], \quad Q=\left[\begin{array}{cc}
0.4567 & 0 \\
0 & 0.0141
\end{array}\right] .
\end{gathered}
$$

Therefore, it follows from Theorem 3.1 that the system (2.3) with given parameters is globally asymptotically stable.

\section{Conclusions}

In this paper, a new delay-dependent asymptotic stability criterion for neural networks with time-varying delay has been proposed. A new class of Lyapunov functional has been introduced to derive some less conservative delay-dependent stability criteria by using the free-weighting matrices method and the technique of dealing with some integral terms. Finally, numerical examples have been given to illustrate the effectiveness of the proposed method.

\section{Acknowledgments}

The authors would like to thank the editors and the reviewers for their valuable suggestions and comments which have led to a much improved paper. This work was supported by Sichuan Provincial Youth Science and Technology Fund (Grant no. 2012JQ0010), Program for New Century Excellent Talents in University (Grant no. NCET-11-1062), National Science Fund for Distinguished Young Scholars of China (Grant no. 51125019), PetroChina Innovation Foundation (Grant no. 2011D-5006-0201), and research on the model and method of parameter identification in reservoir simulation under Grant PLN1121.

\section{References}

[1] J. Cao and L. Wang, "Exponential stability and periodic oscillatory solution in BAM networks with delays," IEEE Transactions on Neural Networks, vol. 13, no. 2, pp. 457-463, 2002.

[2] S. Arik, "Global asymptotic stability of a larger class of neural networks with constant time delay," Physics Letters, Section A, vol. 311, no. 6, pp. 504-511, 2003.

[3] J. Cao and J. Wang, "Global asymptotic and robust stability of recurrent neural networks with time delays," IEEE Transactions on Circuits and Systems I, vol. 52, no. 2, pp. 417-426, 2005.

[4] C. Hua, C. Long, and X. Guan, "New results on stability analysis of neural networks with timevarying delays," Physics Letters, Section A, vol. 352, no. 4-5, pp. 335-340, 2006.

[5] Y. He, M. Wu, and J. H. She, "Delay-dependent exponential stability of delayed neural networks with time-varying delay," IEEE Transactions on Circuits and Systems II, vol. 53, no. 7, pp. 553-557, 2006.

[6] Y. He, G. Liu, and D. Rees, "New delay-dependent stability criteria for neural networks with yimevarying delay," IEEE Transactions on Neural Networks, vol. 18, no. 1, pp. 310-314, 2007. 
[7] T. Li, W. X. Zheng, and C. Lin, "Delay-slope-dependent stability results of recurrent neural networks," IEEE Transactions on Neural Networks, vol. 22, no. 12, part 1, pp. 2138-2143, 2011.

[8] Y. He, G. P. Liu, D. Rees, and M. Wu, "Stability analysis for neural networks with time-varying interval delay," IEEE Transactions on Neural Networks, vol. 18, no. 6, pp. 1850-1854, 2007.

[9] J. Sun, G. P. Liu, J. Chen, and D. Rees, "Improved stability criteria for neural networks with timevarying delay," Physics Letters, Section A, vol. 373, no. 3, pp. 342-348, 2009.

[10] S. Mou, H. Gao, J. Lam, and W. Qiang, "A new criterion of delay-dependent asymptotic stability for Hopfield neural networks with time delay," IEEE Transactions on Neural Networks, vol. 19, no. 3, pp. 532-535, 2008.

[11] L. Hu, H. Gao, and P. Shi, "New stability criteria for Cohen-Grossberg neural networks with time delays," IET Control Theory and Applications, vol. 3, no. 9, pp. 1275-1282, 2009.

[12] R. Yang, H. Gao, and P. Shi, "Novel robust stability criteria for stochastic Hopfield neural networks with time delays," IEEE Transactions on Systems, Man, and Cybernetics, Part B, vol. 39, no. 2, pp. 467474, 2009.

[13] H. Zhang, Z. Liu, G. B. Huang, and Z. Wang, "Novel weighting-delay-based stability criteria for recurrent neural networks with time-varying delay," IEEE Transactions on Neural Networks, vol. 21, no. 1, pp. 91-106, 2010.

[14] S. P. Xiao and X. M. Zhang, "New globally asymptotic stability criteria for delayed cellular neural networks," IEEE Transactions on Circuits and Systems II, vol. 56, no. 8, pp. 659-663, 2009.

[15] X. M. Zhang and Q. L. Han, "New Lyapunov-Krasovskii functionals for global asymptotic stability of delayed neural networks," IEEE Transactions on Neural Networks, vol. 20, no. 3, pp. 533-539, 2009.

[16] Y. Zhang, D. Yue, and E. Tian, "New stability criteria of neural networks with interval time-varying delay: a piecewise delay method," Applied Mathematics and Computation, vol. 208, no. 1, pp. 249-259, 2009.

[17] J. H. Park and O. M. Kwon, "Further results on state estimation for neural networks of neutral-type with time-varying delay," Applied Mathematics and Computation, vol. 208, no. 1, pp. 69-75, 2009.

[18] R. Samli and S. Arik, "New results for global stability of a class of neutral-type neural systems with time delays," Applied Mathematics and Computation, vol. 210, no. 2, pp. 564-570, 2009.

[19] J. Tian and X. Zhou, "Improved asymptotic stability criteria for neural networks with interval timevarying delay," Expert Systems with Applications, vol. 37, no. 12, pp. 7521-7525, 2010.

[20] O. M. Kwon, J. H. Park, and S. M. Lee, “On robust stability for uncertain neural networks with interval time-varying delays," IET Control Theory and Applications, vol. 2, no. 7, pp. 625-634, 2008.

[21] Y. Chen and Y. Wu, "Novel delay-dependent stability criteria of neural networks with time-varying delay," Neurocomputing, vol. 72, no. 4-6, pp. 1065-1070, 2009.

[22] Q. Song, Z. Zhao, and Y. Li, "Global exponential stability of BAM neural networks with distributed delays and reaction-diffusion terms," Physics Letters, Section A, vol. 335, no. 2-3, pp. 213-225, 2005.

[23] S. Arik, "Global asymptotic stability of hybrid bidirectional associative memory neural networks with time delays," Physics Letters, Section A, vol. 351, no. 1-2, pp. 85-91, 2006.

[24] X. Liao, G. Chen, and E. N. Sanchez, "Delay-dependent exponential stability analysis of delayed neural networks: an LMI approach," Neural Networks, vol. 15, no. 7, pp. 855-866, 2002.

[25] L. Hu, H. Gao, and W. X. Zheng, "Novel stability of cellular neural networks with interval timevarying delay," Neural Networks, vol. 21, no. 10, pp. 1458-1463, 2008.

[26] S. Xu and J. Lam, "A new approach to exponential stability analysis of neural networks with timevarying delays," Neural Networks, vol. 19, no. 1, pp. 76-83, 2006.

[27] H. Zhao and J. Cao, "New conditions for global exponential stability of cellular neural networks with delays," Neural Networks, vol. 18, no. 10, pp. 1332-1340, 2005.

[28] Z. Wang, Y. Liu, M. Li, and X. Liu, "Stability analysis for stochastic Cohen-Grossberg neural networks with mixed time delays," IEEE Transactions on Neural Networks, vol. 17, no. 3, pp. 814-820, 2006.

[29] T. Li, L. Guo, C. Sun, and C. Lin, "Further results on delay-dependent stability criteria of neural networks with time-varying delays," IEEE Transactions on Neural Networks, vol. 19, no. 4, pp. 726-730, 2008.

[30] C. D. Zheng, H. Zhang, and Z. Wang, "New delay-dependent global exponential stability criterion for cellular-type neural networks with time-varying delays," IEEE Transactions on Circuits and Systems II, vol. 56, no. 3, pp. 250-254, 2009.

[31] C. D. Zheng, L. B. Lu, and Z. S. Wang, "New LMT-based delay-dependent criterion for global asymptotic stability of cellular neural networks," Neurocomputing, vol. 72, no. 13-15, pp. 3331-3336, 2009.

[32] Z.-G. Wu, J.H. Park, H. Su, and J. Chu, “New results on exponential passivity of neural networks with time-varying delays," Nonlinear Analysis, vol. 13, no. 4, pp. 1593-1599, 2012. 
[33] G. Zhang, T. Li, and S. Fei, "Further stability criterion on delayed recurrent neural networks based on reciprocal convex technique," Mathematical Problems in Engineering, vol. 2012, Article ID 829037, 14 pages, 2012.

[34] Y. Ge, T. Li, and S. Fei, "Master-slave synchronization of stochastic neural networks with mixed timevarying delays," Mathematical Problems in Engineering, vol. 2012, Article ID 730941, 18 pages, 2012.

[35] H. Wu, N. Li, K. Wang, G. Xu, and Q. Guo, "Global robust stability of switched interval neural networks with discrete and distributed time-varying delays of neural type," Mathematical Problems in Engineering, vol. 2012, Article ID 361871, 18 pages, 2012.

[36] H. Zhang, T. Li, and S. Fei, "Synchronization for an array of coupled cohen-grossberg neural networks with time-varying delay," Mathematical Problems in Engineering, vol. 2011, Article ID 831695, 22 pages, 2011.

[37] Y. Li and K. Zhao, "Robust stability analysis of fuzzy neural network with delays," Mathematical Problems in Engineering, vol. 2009, Article ID 826908, 13 pages, 2009.

[38] S. Boyd, V. Balakrishnan, E. Feron, and L. El Ghaoui, Linear Matrix Inequalities in Systems and Control, SIMA, Philadelphia, Pa, USA, July 1994. 


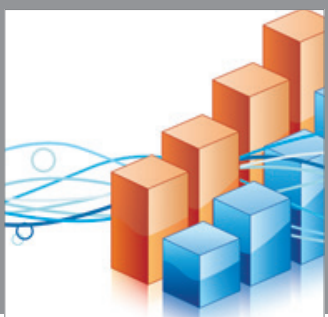

Advances in

Operations Research

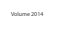

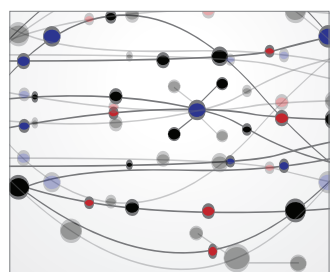

\section{The Scientific} World Journal
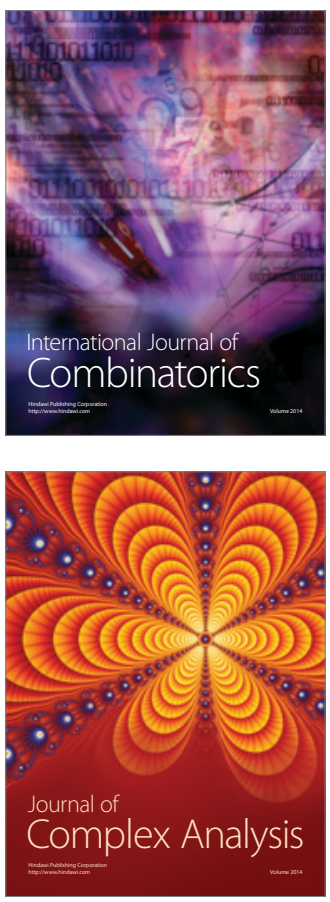

International Journal of

Mathematics and

Mathematical

Sciences
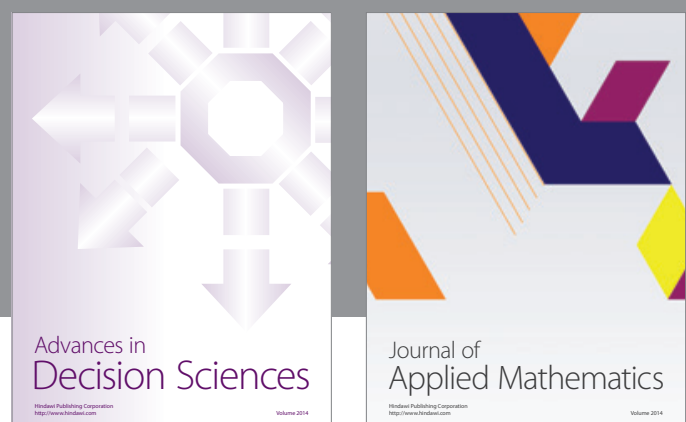

Journal of

Applied Mathematics
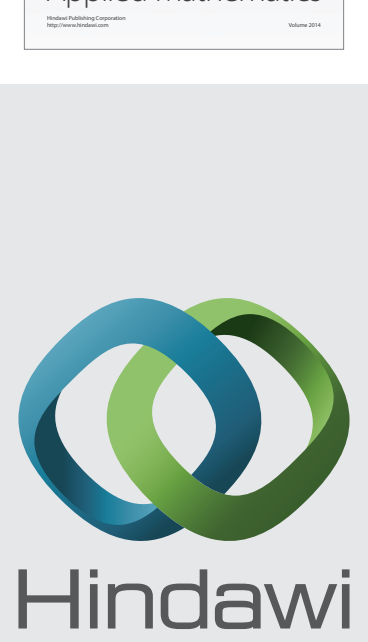

Submit your manuscripts at http://www.hindawi.com
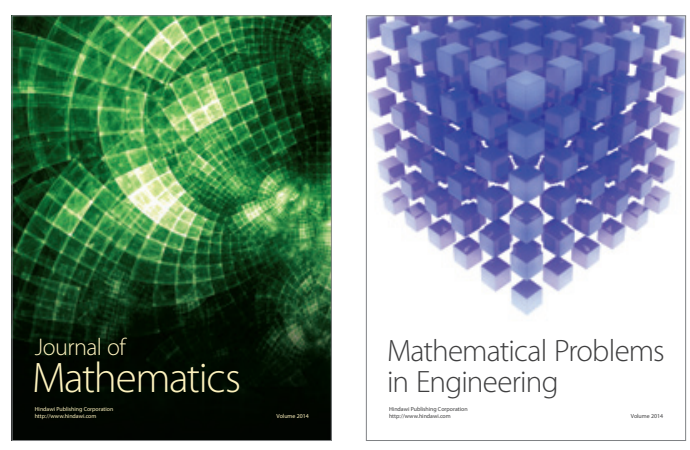

Mathematical Problems in Engineering
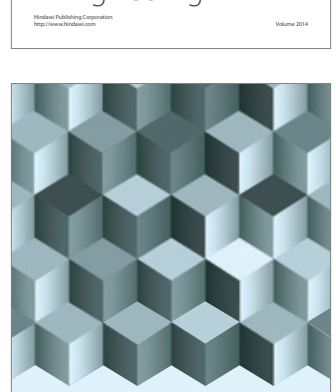

Journal of

Function Spaces
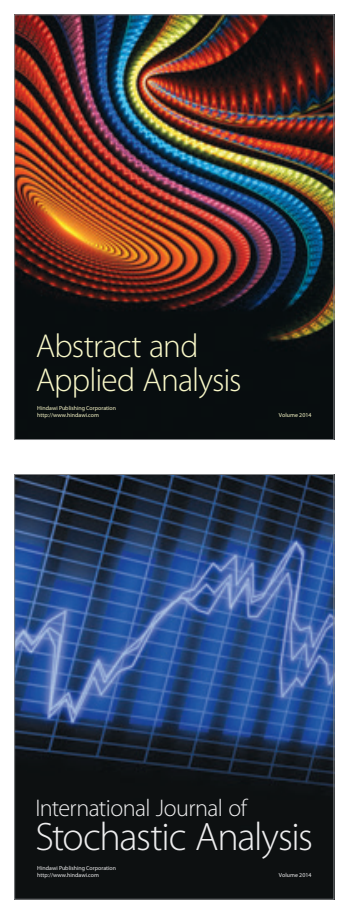

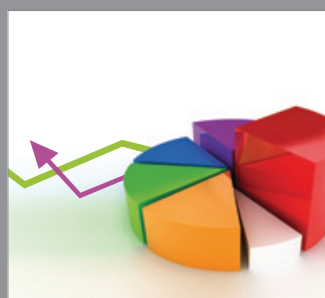

ournal of

Probability and Statistics

Promensencen
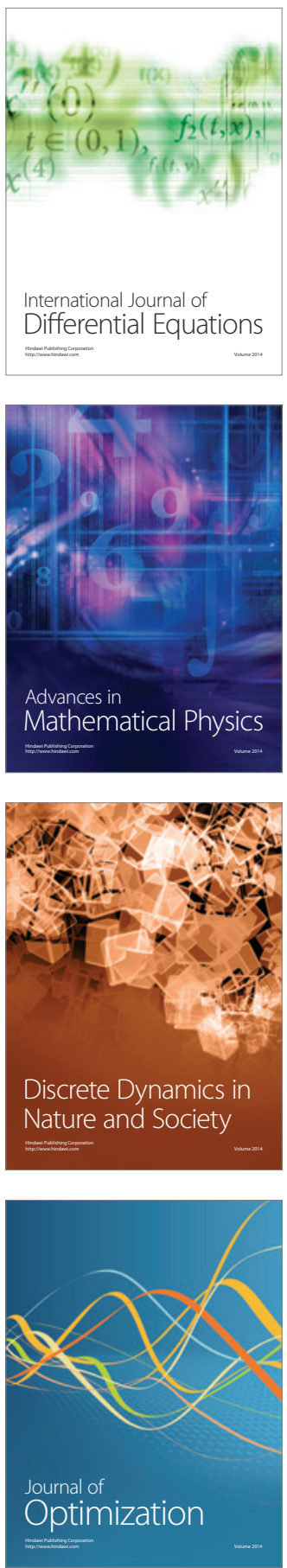